\title{
UNA HISTORIA DE LA MEDICINA FAMILIAR MEXICANA
}

\section{A HISTORY OF MEXICAN FAMILY MEDICINE}

\section{Homero De los Santos-Reséndiz*}

Departamento de Investigación, Facultad de Medicina, Universidad Autónoma de Durango, campus Saltillo, Coahuila, México

RESUMEN: La Revista Mexicana de Medicina Familiar se editó por primera vez en el año 1987. Fue la Asociación Nacional de Residentes y Especialistas en Medicina Familiar AC, fundadora y poseedora de los derechos de autor, quien cedió al Colegio Mexicano de Medicina Familiar AC el número internacional normalizado de publicaciones seriadas (ISSN por sus siglas en inglés), con el único interés de promover el desarrollo de los especialistas en medicina familiar, y así se hizo posible reeditar con el nombre original lo que sería su órgano oficial de difusión a partir del año 2014. Ahora, con la incorporación de la Revista Mexicana de Medicina Familiar en el catálogo de revistas médicas de LATINDEX, parece oportuno reconocer a aquellos médicos y agrupaciones de medicina familiar que han contribuido en este logro, y a la vez reseñar una historia de éxito de la medicina familiar mexicana. Las publicaciones en medicina familiar en México son escasas, por lo que este documento y esta publicación electrónica representa una oportunidad maravillosa para compartir nuevos conocimientos y experiencias mediante una revista médica de alta calidad científica, con la esperanza de que llegue a significar para las nuevas generaciones un legado de esfuerzo, de conocimientos con el nivel de experto en la materia, de capacidad de organización y, sobre todo, de ese espíritu de superación que debiera existir en todos los especialistas en medicina familiar, tan necesario en los momentos de cambio constante que vive la profesión médica.

Palabras clave: Medicina familiar. Historia. Indización.
ABSTRACT: The Mexican Journal of Family Medicine was published for the first time in 1987. It was the National Association of Residents and Specialists in Family Medicine AC, founder and holder of the copyright, who ceded to the Mexican College of Family Medicine AC the International Standard of Serial Numbers (ISSN), with the sole interest of promoting the development of family medicine specialists, and thus it was possible to reissue with the original name what would be its official dissemination body as of 2014. Now, with the incorporation of the Revista Mexicana de Medicina Familiar in the catalog of medical journals of LATINDEX, it seems appropriate to recognize those specialists and family medicine groups that have contributed to this achievement and at the same time, review a success story of Mexican family medicine. Publications in family medicine in Mexico are scarce, so this document and this electronic publication represents a wonderful opportunity to share new knowledge and experiences through a medical journal of high scientific quality, with the hope that it will mean for the new generations a legacy of effort, knowledge with the level of expert in the field, organizational capacity and above all, that spirit of improvement that should exist in all specialists in family medicine, so necessary in times of constant change that lives the medical profession.

Key words: Family medicine. History. Indexation.

\section{Correspondencia:}

*Homero De los Santos-Reséndiz

E-mail: hosare11@ hotmail.com
Fecha de recepción: 09-10-2019

Fecha de aceptación: 10-12-2019
Disponible en internet: 08-04-2020

Rev Mex Med Fam. 2020;7:38-41 DOI: 10.24875/RMF.19000227

2007-9710 / @ 2019 Federación Mexicana de Especialistas y Residentes en Medicina Familiar. Publicado por Permanyer. Este es un artículo open access bajo la licencia CC BY-NC-ND (http://creativecommons.org/licenses/by-nc-nd/4.0/). 
Los médicos especialistas en medicina familiar (MF) estamos de plácemes por la incorporación de la Revista Mexicana de Medicina Familiar (RMMF), órgano de difusión del Colegio Mexicano de Medicina Familiar (COLMEXMF), en el Índice Latinoamericano de Publicaciones Científicas (LATINDEX), que es «el sistema regional de información en línea para revistas científicas de América Latina, el Caribe, España y Portugal, cuyo compromiso es registrar y ayudar a mejorar la calidad de la edición de revistas científicas y, a la vez, garantizar una mayor visibilidad de la producción científica iberoamericana poniendo la información recopilada en línea, con acceso libre, en un portal dedicado» ${ }^{1}$. El logro alcanzado ha sido posible gracias a todos los MF que con su lectura hacen realidad sueños, propósitos y objetivos de otros MF, quienes con enorme esfuerzo y dedicación dan orden a una praxis cada vez más original, siempre con fundamentos científicos, y que la transmiten por escrito de manera desinteresada, todos encaminados a alcanzar la misión: el desarrollo de la especialidad de medicina familiar y de los MF.

Mención aparte merecen aquellos que de una $\mathrm{u}$ otra forma han participado en la organización y elaboración de la RMMF, pues quien se anima a hacerlo establece un fuerte compromiso, porque el trabajo es arduo y requiere conocimientos propios sobre la materia, que solo con el tiempo y estar cumpliendo la tarea de editar el siguiente número se pueden lograr. El COLMEXMF ha tenido a bien reconocer la labor y el empeño del Dr. Med. José Manuel Ramírez Aranda, actual editor de la RMMF, por elevar el nivel científico de esta publicación, ahora certificado por un organismo internacional que la coloca en su catálogo de revistas médicas. Alcanzar esta meta, la primera en ese largo proceso de calidad por medio de la mejora continua, refleja el espíritu que tienen los MF por mantener siempre su superación profesional, además de su capacidad de organización y su expertise en la atención médica primaria. Ante este momento, que con justa razón pudiera decirse memorable para los MF mexicanos, me parece de interés ampliar la información sobre el origen y evolución de la RMMF, y así rendir un pequeño homenaje a todos esos colegas visionarios, que seguramente estarían muy satisfechos de saber que su sueño está cada vez más cerca de convertirse en realidad.

Fue en el año 1987 cuando un grupo de MF de la Asociación Nacional de Residentes en Medicina Familiar AC (ANREMFAC), encabezados como editor por el Dr. Juan Sergio Rivera Escamilla, tuvieron la iniciativa y lograron por primera vez editar la RMMF. Su edición fue en hojas de papel bond de diferentes colores: azul, verde o amarillo. La impresión se hizo con mimeógrafo, lo que requería llevar a cabo el «picado» de las hojas, colocar estas en el rodillo e impregnarlas de tinta que casi siempre se extendía a manos, rostro y ropa de quien llevaba a cabo esta acción. Posteriormente, cada una de las hojas impresas se ordenaban y grapaban por la parte de en medio, se doblaban y así se le daba forma a la RMMF. Un bonito trabajo artesanal fue el que realizó este grupo de MF, llegando a producir un tiraje hasta de 500 ejemplares. Junto con Juan Sergio, destacan la participación de Arturo Cerda Ocho, quien colaboró para sentar las bases filosóficas de la práctica en medicina familiar mexicana como una especialidad médica y además tuvo una importante participación en el comité editorial junto con Víctor Chávez Aguilar, María del Carmen García Peña, Ricardo Anzures Carro, Octavio Pons Álvarez, Tomás Chapa Luque y Sofía Villa Contreras, entre otros. Participaron con colaboraciones propias muchos médicos y residentes en temas de interés para el ejercicio de la especialidad, desde clínicos hasta educativos, de administración médica y, sobre todo, relacionados con la estructura y funcionalidad de la familia mexicana. La vida de la RMMF fue corta en esta primera etapa. A pesar del interés que despertó su edición, no fueron más de 13 números los que llegaron a hacerse realidad en sus casi 5 años de existencia, pues dejó de editarse en 1991.

Durante la gestión 2013-2015 del COLMEXMF se tomó la decisión, obviamente 
colegiada, de llevar a cabo el proyecto de reedición de la RMMF. Ricardo Anzures Carro y María del Carmen García Peña fueron los líderes y editores de este proyecto. La primera tarea fue obtener el nombre para la revista, el cual le pertenecía a la ANREMFAC, pues fue esta quien realizó los registros correspondientes ante las autoridades establecidas por la Ley de Imprenta para obtener el denominado ISSN en la Dirección General de los Derechos de Autor. Gracias a la cesión de los derechos de autor de la ANREMFAC, ahora denominada Asociación de Médicos Familiares y Residentes de Medicina Familiar (AMREFAC), con Luis García Rey al frente, se convirtió en realidad poder asignar el nombre de RMMF a la nueva publicación y designarla como el órgano oficial de difusión del COLMEXMF. La visión que se tuvo al momento de arrancar el proyecto fue llegar a editar una revista médica calificada con la calidad suficiente para ser indizada en los catálogos de revistas médicas de mayor calidad nacional e internacional. Para eso se realizaron las acciones necesarias para sentar las bases legales ante cualquier autoridad (Secretaría de Educación Pública [SEP], Secretaría de Hacienda y Crédito Público [SHCP], Registro Público de la Propiedad) con el fin de cumplir con los requisitos establecidos para llegar a la meta, entre los que se encontraba también la necesidad de publicar la RMMF impresa y no únicamente en forma electrónica, lo que implicó una importante erogación económica en diseñadores, correctores de texto, impresos de alta calidad y trabajo secretarial. El primer consejo editorial se integró con José Manuel Ramírez Aranda, Sergio Arturo Juárez Márquez, Fabián Ávalos Pérez, Cidronio Albavera Hernández, Haydee Cabrera Reyes, Carlos Alberto Durán Muñoz, Yolanda García Valerio, Rosalba Morales Jaimes, Victoria Pineda Aquino, Octavio Noel Pons Álvarez, María de Lourdes Rojas Armadillo, Sergio Sánchez García, Elizabeth Soler Huerta, José Fernando Torres Sánchez y Homero de los Santos Reséndiz. El diseño del logotipo de la revista lo realizó Aurelio García
Cortés, mientras que el diseño de la página web de la revista estuvo a cargo de Alejandro Diep Montiel y la edición la efectuó Martha Baranda Torres.

El trabajo previo, necesario para la reedición de la RMMF, llevó aproximadamente 8 meses, en los que se tuvieron que sortear diferentes situaciones, unas relacionadas con la poca experiencia de todo el grupo editorial, otras al elaborar los requisitos para autores, también con el formato para cumplir con criterios de las revistas médicas de alta calidad, en la definición de las secciones y en sí, con todo el trabajo operativo y normativo para hacer posible esto, que presentaba todas las características de una utopía, pues la meta ahí estaba, pero cada vez que se creía alcanzada, esta se alejaba y, hasta la fecha, se sigue alejando de manera infinita. Este gran reto se pudo afrontar de manera positiva gracias a la asesoría otorgada por editores de otras revistas médicas con amplia experiencia, como la Revista Médica del Instituto Mexicano del Seguro Social (IMSS), entre otras. Respondieron de manera puntual a las dudas de los editores, sobre todos en esos detalles que toda publicación médica requiere: el número del tiraje ideal, «¡cuidado! así no va», "no se pone entrecomillado en esa parte», el encabezado indispensable en cada hoja con todas sus características, el número de páginas conveniente, los puntos y las comas, etcétera.

La RMMF, una publicación cuatrimestral, lanzó el volumen 1, número 1, enero-abril 2014, en el marco del XXVI Congreso Nacional de Medicina Familiar que se realizó en Juriquilla, Querétaro. Cuando el Dr. Guillermo Soberón Acevedo, ex Secretario de Salud de México, supo de la RMMF, expresó con gran júbilo: «ese es el verdadero estatus que debe buscar y tener el especialista en medicina familiar, pues cuenta con un verdadero cuerpo de conocimientos que debe desarrollar con investigación propia, donde ponga a prueba su papel y su intervención sustantiva para lograr calidad médica de excelencia». El Dr. Carlos Ernesto Varela Rueda, fundador del primer curso de especialización en Medicina Familiar 
del IMSS en 1971, coincide con esa opinión y agrega: «Vaya, hasta que pudieron hacerlo, pero recuerden que aunque es importante la publicación del número 1 , lo más difícil será mantener su publicación y probar que la especialidad de medicina familiar es en verdad un cuerpo de conocimientos que puede modificar la realidad de cualquier problema de salud de la persona y su familia».

Las asociaciones, sociedades y colegios de medicina familiar de los diferentes Estados del país, que en aquel entonces constituían el COLMEXMF, tuvieron, y siguen teniendo, una participación fundamental. Fueron promotores entre sus afiliados para contar con la materia prima de la RMMF: artículos originales, ensayos, etc., elemento nuclear en esta publicación, pues si el objetivo era transmitir la experiencia de los $\mathrm{MF}$, el contenido de la revista tenía que provenir de los mismos MF, aunque además se consideraron, y de manera importante, a los residentes y profesores de MF como autores y lectores; también contribuyeron con otras acciones estratégicas, como la promoción y suscripciones a la revista, pero sobre todo fueron los mensajeros de la RMMF, pues por medio de estas agrupaciones de MF se hacía llegar a cada lector el ejemplar correspondiente; posteriormente, al adquirir la franquicia postal, se les pudo liberar de esa función.

Las siguientes gestiones directivas del COLMEXMF, a cargo de Carlos Adolfo Martínez Rentería, José Luis Huerta González y Fernando Hernández Ávila, supieron sortear todas las dificultades que tiene la edición de cada nuevo número y han podido conducir el proyecto en la ruta establecida desde su fundación, con resultados tangibles en calidad científica y de edición, en el proceso de mejora continua, que se manifiesta con la incorporación de la RMMF al mundo de las redes sociales, que en esta era digital es igual a darle «voz» al MF mexicano con difusión nacional e internacional. Aunado a lo anterior, en esta nueva etapa de la RMMF el actual consejo editorial está integrado con MF mexicanos e internacionales, de reconocido prestigio en México, Canadá, EE.UU., Centroamérica y Sudamérica, lo que ha venido a fortalecer, no solo su contenido, sino también y de manera exponencial, su presencia y difusión.

Como se puede notar, hablar de impresos, mimeógrafo, hojas esténcil, tinta, calidad del papel, franquicia postal, nos habla de la antigüedad de la RMMF, pues su historia se inicia hace 33 años. Después de pasar 23 años adormecida, los últimos 5 años se han caracterizado por su renacimiento y desarrollo consolidado. La lectura se ha desplazado del texto impreso en papel a textos digitales, un mundo digital donde la transmisión de mensajes parece accesible a cualquier ser humano del mundo, un mundo al que ha arribado de manera exitosa la RMMF. Ante esto, no queda más que terminar esta breve historia de la siguiente manera:

¡Felicidades a los MF mexicanos por mantener y mejorar, cada vez más, la edición de la RMMF, órgano oficial de difusión del COLMEXMF!

\section{AGRADECIMIENTOS}

A Ricardo Anzures Carro, por la valiosa información proporcionada para el presente artículo.

\section{COMENTARIOS}

El autor declara haber sido Presidente del Colegio Mexicano de Medicina Familiar en la gestión 2013-2015, ahora FedMexMF.

\section{FINANCIAMIENTO}

La presente investigación no ha recibido ayudas específicas provenientes de agencias del sector público, sector comercial o entidades sin ánimo de lucro.

\section{CONFLICTO DE INTERESES}

Ninguno.

\section{BIBLIOGRAFÍA}

\footnotetext{
1. Flores A, Penkova S, Adelaida Román A. Once años de LATINDEX: una experiencia al servicio de las publicaciones científicas iberoamericanas. Simbiosis. 2009;6(1):1548-3436.
} 